\title{
Síndrome de Hedinger
}

\author{
Hedinger syndrome
}

\author{
Ronald E. Rivas-Gálvez ${ }^{*}$, Luis E. Estrada-Martínez¹, Eliana Yagual-Gutiérrez², Luz D. Sandoval-Castillo³ \\ y Julieta D. Morales-Portano 4 \\ ${ }^{1}$ Cardiología Clínica Adultos; ${ }^{2}$ Cirugía Cardiovascular Adultos; ${ }^{3}$ Servicio de Imagen Cardiovascular; ${ }^{4}$ Servicio de Cardiología y Ecocardiografía. \\ Centro Médico Nacional 20 de Noviembre, Instituto de la Seguridad Social de los Trabajadores del Estado, Ciudad de México, México
}

\section{Resumen}

La afectación cardíaca por tumor carcinoide (síndrome de Hedinger) sigue siendo una causa importante de morbilidad y mortalidad entre los pacientes con síndrome carcinoide y tumores neuroendocrinos metastásicos. El estudio de imagen inicial ante la sospecha clínica es el ecocardiograma transtorácico, el cual es importante para la detección temprana de la afectación cardíaca. La cirugía cardíaca, en casos apropiados, es la única terapia definitiva para el tumor carcinoide con afectación cardíaca y mejora los síntomas del paciente y la supervivencia. El tratamiento es complejo y multidisciplinario; el estado cardíaco, el síndrome hormonal y la carga tumoral son fundamentales para guiar el momento óptimo de la cirugía. Se presenta el caso de una paciente joven, con síndrome de Hedinger con afectación valvular tricúspide y pulmonar, quien recibió tratamiento quirúrgico y tuvo evolución exitosa.

Palabras clave: Tumor carcinoide. Estenosis pulmonar. Estenosis de la válvula tricúspide.

\begin{abstract}
Cardiac carcinoid tumor involvement (Hedinger's syndrome) remains a major cause of morbidity and mortality among patients with carcinoid syndrome and metastatic neuroendocrine tumors. The initial imaging study in case of clinical suspicion is the transthoracic echocardiogram, which is important for the early detection of cardiac involvement. Cardiac surgery, in appropriate cases, is the only definitive therapy for carcinoid tumor with cardiac involvement and improves patient symptoms and survival. Treatment is complex and multidisciplinary; cardiac status, hormonal syndrome, and tumor burden are essential to guide the optimal timing of surgery. The case of a young female patient with Hedinger's syndrome with tricuspid and pulmonary valve involvement, who received surgical treatment and had a successful evolution.
\end{abstract}

Keywords: Carcinoid tumor. Pulmonary stenosis. Tricuspid valve stenosis.

\section{Introducción}

Los tumores carcinoides son parte de una categoría mayor de tumores, conocidos como tumores neuroendocrinos; son de crecimiento lento y tienen potencial significativo para producir metástasis hepáticas y liberar cantidades excesivas de aminas vasoactivas en la circulación sistémica ${ }^{1}$. Aunque el progreso en el manejo médico y quirúrgico de los pacientes con tumores

\section{Correspondencia:}

*Ronald Rivas-Gálvez

E-mail: rivasgalvez3007@gmail.com
Disponible en internet: 22-02-2022 Rev Colomb Cardiol. 2022;29(1):94-99 www.rccardiologia.com 0120-5633 / C 2021 Sociedad Colombiana de Cardiología y Cirugía Cardiovascular. Publicado por Permanyer. Este es un artículo open access bajo la licencia CC BY-NC-ND (http://creativecommons.org/licenses/by-nc-nd/4.0/). 
neuroendocrinos metastásicos ha conducido en una mejoría de los síntomas y de la supervivencia, la afectación cardíaca por tumor carcinoide sigue siendo una causa importante de morbilidad y mortalidad. Entre los pacientes con síndrome carcinoide se ha descrito una afectación cardíaca en el $20 \%$ al $50 \%$ de los casos $^{2}$. Se ha observado un ligero predominio masculino, de aproximadamente el $60 \%$, con una edad media al momento del diagnóstico de $59 \pm 11$ años $^{3}$. Sin tratamiento, el pronóstico de la enfermedad coronaria es pobre, con una supervivencia a 3 años tan baja como del $31 \%$, en comparación con el $68 \%$ en los pacientes con tumores neuroendocrinos sin afectación cardíaca. La enfermedad cardíaca carcinoide con síntomas avanzados (clase funcional III o IV de la New York Heart Association [NYHA]) conlleva particularmente un mal pronóstico, con una mediana de supervivencia de solo 11 meses. Sin embargo, en las últimas décadas, el pronóstico de los pacientes con enfermedad cardíaca carcinoide ha mejorado. En una serie retrospectiva de 200 pacientes con síndrome carcinoide y enfermedad cardíaca carcinoide, la supervivencia media mejoró de 1.5 años en los años 1980 a 4.4 años a fines de los años 1990, y los datos sugieren que esta mejoría está relacionada con el aumento de las tasas de cirugía cardíaca y el uso de análogos de la somatostatina ${ }^{2-4}$.

\section{Caso clínico}

Paciente de sexo femenino, de 21 años de edad, sin antecedentes médicos quirúrgicos, que presenta 5 años de evolución de alopecia circular eventual, astenia, fatiga, adinamia y diarrea crónica, refractaria a tratamiento. En los últimos 3 meses presentó edema de miembros inferiores, disnea a esfuerzos moderados, episodios de eritema en la cara, el tronco y las extremidades, artralgias de manos y pies, astenia, adinamia y pérdida de peso. En la exploración física presentó episodios de enrojecimiento, con evidencia de eritema malar (Fig. 1), cuello con ingurgitación yugular, reflujo hepatoyugular, soplo holosistólico en foco tricúspide que aumentó con la maniobra de Rivero-Carvallo, soplo holosistólico en foco pulmonar de grado IV/IV, S3 y ritmo de galope; en el abdomen se detectó hepatomegalia sin dolor a la palpación, y presentaba edema en las extremidades inferiores con signo de Godet. En los exámenes de laboratorio se evidenciaron concentraciones de serotonina de $397 \mathrm{ng} / \mathrm{ml}$ y ácido 5 -hidroxiindolacético en orina de 24 horas de $95 \mathrm{mg}$. Se realizó tomografía computarizada abdominal trifásica (Fig. 2), la cual reportó múltiples metástasis hipervasculares en

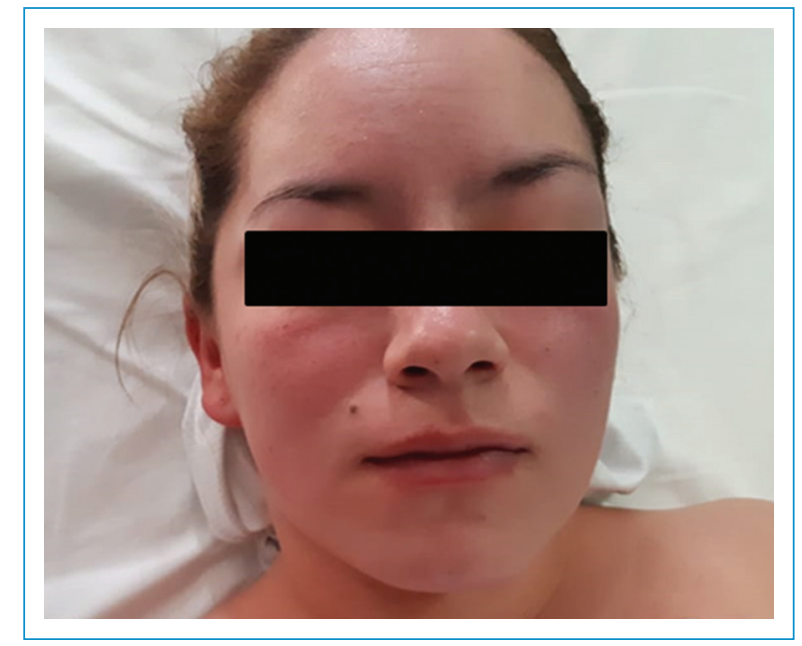

Figura 1. Paciente antes de la intervención quirúrgica.

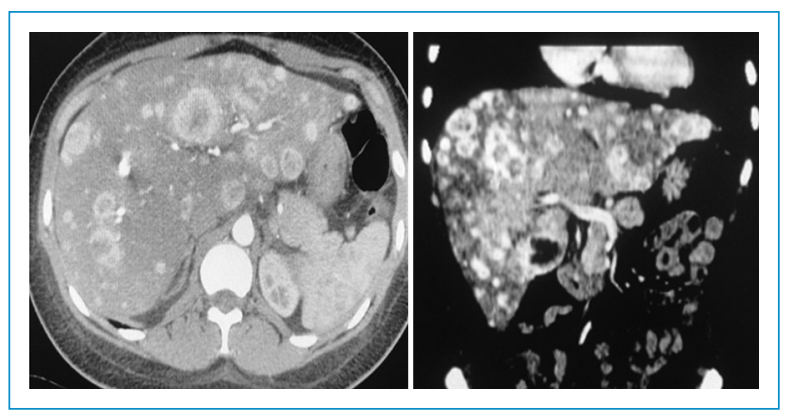

Figura 2. Tomografía computarizada de abdomen en la que se evidencian lesiones metastásicas en el hígado.

el hígado, por lo que, como primera posibilidad, se consideró tumor carcinoide neuroendocrino, con lesión sospechosa de primario en la región ampular, con ganglios retroperitoneales inespecíficos. Se inició tratamiento con lanreotide, $120 \mathrm{mg}$ por vía subcutánea mensual. Posteriormente fue referida a cardiología, donde se le realizó ecocardiograma transtorácico y transesofágico (Fig. 3). El caso presenta las siguientes lesiones: 1) Doble lesión tricúspide: insuficiencia masiva y estenosis grave y 2) doble lesión pulmonar: insuficiencia grave y estenosis grave. Se realizó resonancia magnética cardíaca (Fig. 4) que reportó válvula tricúspide con engrosamiento y retracción de sus valvas, doble lesión con predominio de la insuficiencia, la cual fue grave, válvula pulmonar con engrosamiento y retracción de sus valvas, y doble lesión con predominio de la estenosis e insuficiencia moderada. Durante la intervención quirúrgica se visualizaron las válvulas 

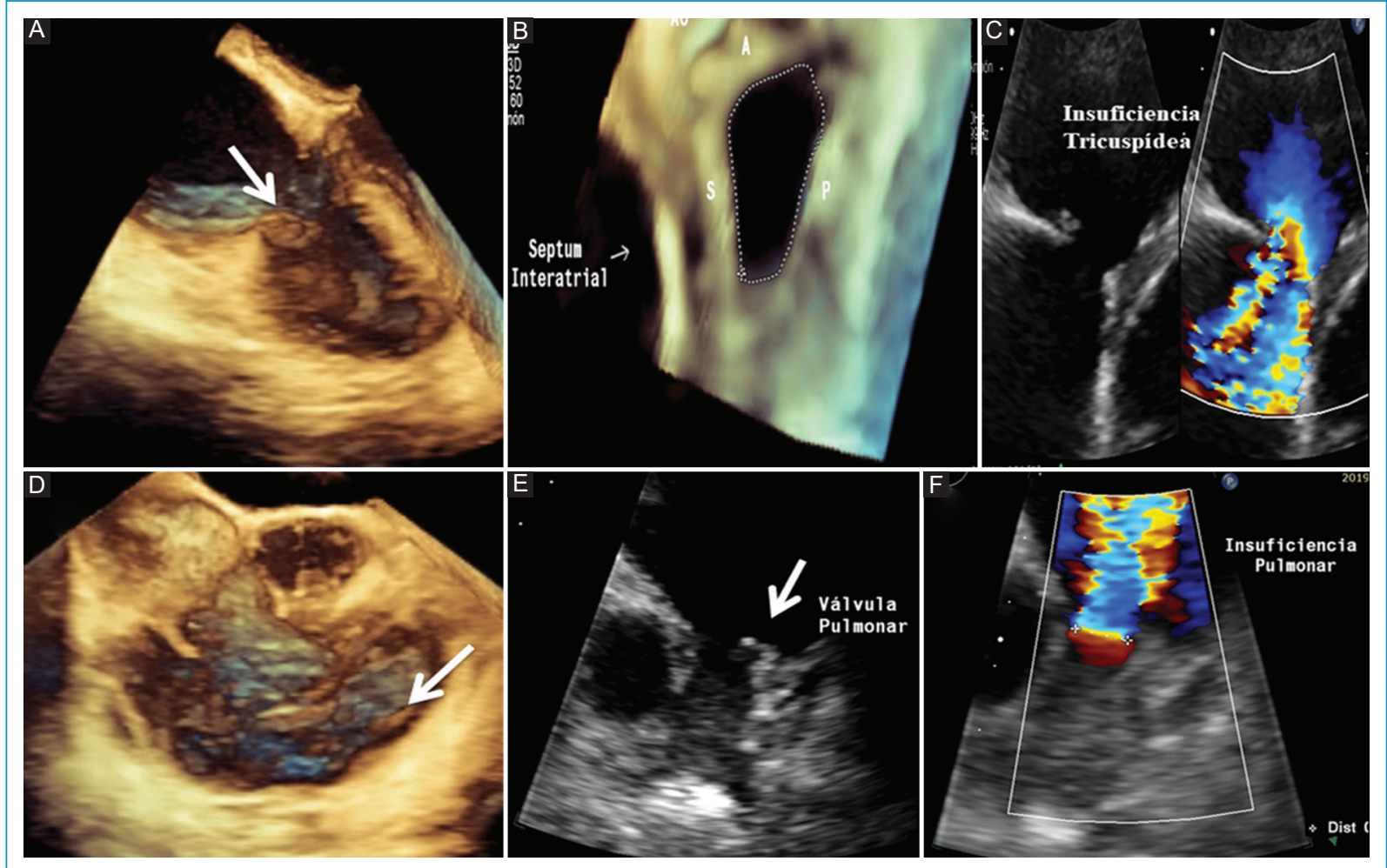

Figura 3. Ecocardiograma transtorácico 3D y 2D de ventrículo derecho y válvula tricúspide, en el que se observa la valva anterior engrosada (flecha). A: restricción del movimiento de tres valvas, el cual condiciona estenosis. B: insuficiencia tricúspide. C: volumen 4D de la vía de entrada y salida del ventrículo derecho, en el que se observan valvas pulmonares engrosadas y retraídas. D: condicionan estenosis. F: condicionan insuficiencia.
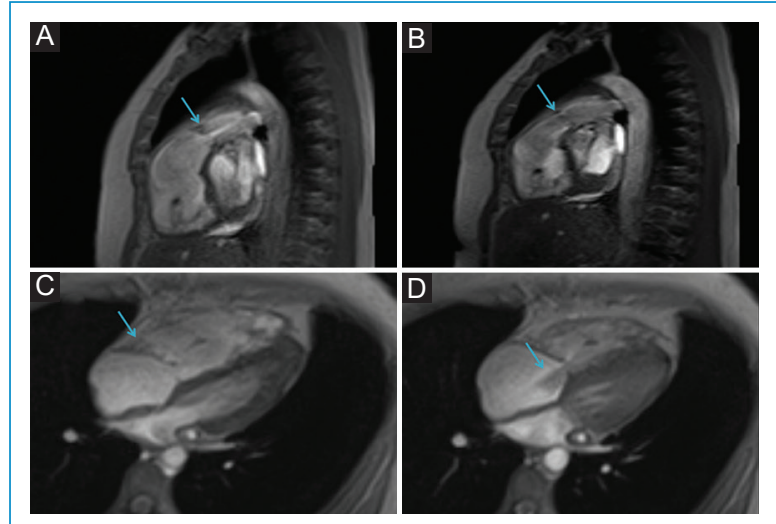

Figura 4. Resonancia magnética cardíaca en secuencia de cine. A: corte sagital del tracto de salida del ventrículo derecho en sístole que muestra la válvula pulmonar engrosada con flujo de aceleración por estenosis. B: corte sagital del tracto de salida del ventrículo derecho en diástole, sin coaptación completa e insuficiencia grave. C: secuencia de cine en cuatro cámaras en diástole con válvula tricúspide engrosada. D: secuencia de cine en cuatro cámaras en sístole con válvula tricúspide engrosada, sin coaptación completa, con jet de insuficiencia. tricúspide y pulmonar (Fig. 5). Se realizó sustitución valvular tricúspide y pulmonar con válvulas biológicas Edwards de $27 \mathrm{~mm}$ y $21 \mathrm{~mm}$, respectivamente. Se reportó biopsia (Fig. 6) y se hizo ecocardiograma de control posquirúrgico, en el que se evidenció mejoría.

\section{Discusión}

Los tumores carcinoides son raros (2.5 a 5.0 casos por 100,000 habitantes/año); son neoplasias neuroendocrinas de crecimiento lento, con potencial significativo para producir metástasis hepáticas y liberar cantidades excesivas de aminas vasoactivas en la circulación sistémica como consecuencia. El sitio primario más común de los tumores carcinoides es el tracto gastrointestinal $(60 \%)$; un tercio de ellos (34\%) surgen en el intestino delgado y hasta el $75 \%$ de estos pueden hacer metástasis al hígado ${ }^{5}$. Hasta el $15 \%$ de los pacientes pueden desarrollar síndrome carcinoide, con manifestaciones de enrojecimiento cutáneo y gastrointestinal, hipermotilidad y afectación cardíaca ${ }^{6}$. Las 


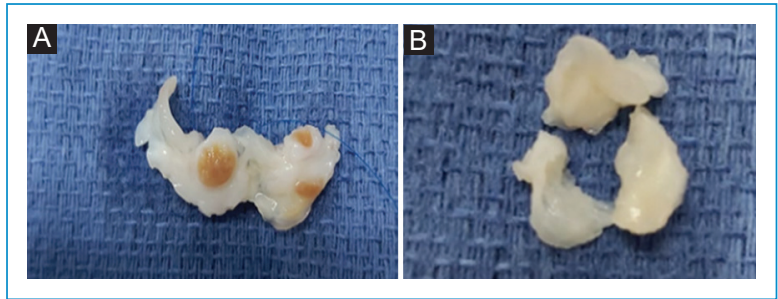

Figura 5. A: válvula tricúspide con valvas acortadas y fibróticas. B: válvula pulmonar trivalva con fibrosis, acortamiento y retracción.

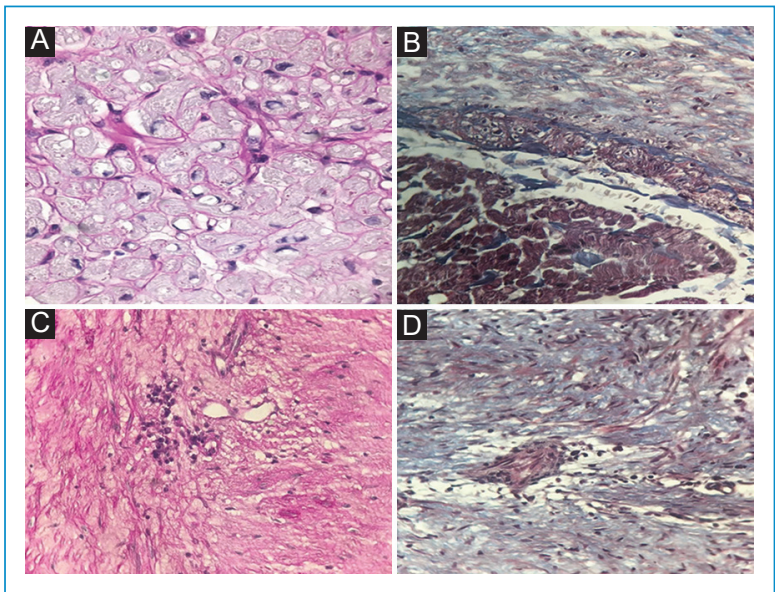

Figura 6. A-D: cortes histológicos de las válvulas pulmonar y tricúspide, en los que se reporta hiperplasia hialina.

manifestaciones cardíacas, también conocidas como enfermedad cardíaca carcinoide, son causadas por la deposición endocárdica de placas fibróticas perladas (notables por la ausencia de elasticidad de las fibras) que, generalmente, se extienden a las válvulas del lado derecho y dan lugar a múltiples patrones de disfunción valvular grave. La formación de placa causa constricción anular, engrosamiento de la valva y fusión del aparato subvalvular, y una marcada degeneración de la arquitectura de la valva que conduce a la retracción grave y a la no adaptación de la válvula, que permanece fija en posición semiabierta ${ }^{6,7}$.

Se cree que la patogenia de la enfermedad cardíaca carcinoide es multifactorial y no se comprende por completo. Se considera que una variedad de sustancias vasoactivas secretadas por el tumor, incluidas serotonina, prostaglandinas, histamina, bradicinina $y$ otras sustancias con propiedades proliferativas de fibroblastos, como las taquicininas (sustancia $P$, neuroquinina $A$, neuropéptido $K$ ) o el factor de crecimiento transformante beta, están involucradas en la enferme$\mathrm{dad}^{8}$. Existe una creciente evidencia que sugiere que la serotonina desempeña un papel importante en la patogenia de la enfermedad cardíaca. Es ampliamente conocido que el ácido 5-hidroxiindolacético urinario, el metabolito de la serotonina, es significativamente más alto en pacientes con enfermedad cardíaca carcinoide, en comparación con aquellos sin compromiso cardía$\mathrm{CO}^{9}$. Entre los hallazgos patológicos de la enfermedad cardíaca carcinoide se encuentran depósitos en forma de placa en el endocardio de las cúspides valvulares, así como en las valvas, las cuerdas tendinosas, los músculos papilares y las cámaras cardíacas, y ocasionalmente dentro de la íntima de las arterias pulmonares o la aorta. Los depósitos están compuestos de miofibroblastos y células musculares lisas rodeadas por componentes de la matriz extracelular (colágeno y matriz mixoide), y cubiertas por una capa de células endoteliales ${ }^{10}$. Las válvulas y el endocardio del lado derecho del corazón son los más afectados, lo cual, generalmente, se debe a la presencia de metástasis hepáticas que secretan grandes cantidades de péptidos vasoactivos que luego llegan al corazón derecho sin ser inactivados. En la válvula pulmonar, esto conduce a la adherencia de las valvas al endotelio de la arteria pulmonar, con la consiguiente insuficiencia, estenosis o ambas. En contraste, la regurgitación tiende a predominar con la enfermedad de la válvula tricúspide debido a la retracción de las valvas (síndrome de Hedinger); las válvulas del lado izquierdo se afectan con menos frecuencia, y se plantea la hipótesis de que se salvan debido a la inactivación de las hormonas vasoactivas por la circulación pulmonar y, así, la afectación del miocardio directamente solo ocurre en el $4 \%$ de los $\operatorname{casos}^{11}$. Los síntomas tempranos de regurgitación tricuspídea grave y aislada incluyen fatiga y disnea de esfuerzo debido a un bajo gasto cardíaco. El edema periférico con congestión hepática y la anorexia consiguiente también pueden ocurrir con la presión auricular derecha elevada. Así mismo, las arritmias auriculares son comunes en el contexto de la ampliación auricular derecha. En la exploración física, el hallazgo más temprano de regurgitación tricuspídea grave suele ser la distensión venosa yugular con una prominente onda $v$ sistólica, aunque su presencia varía entre el $35 \%$ y el $75 \%$ de los pacientes. Otros hallazgos pueden incluir un impulso ventricular derecho palpable y soplos tricúspides, además de regurgitación pulmonar ${ }^{11}$.

Entre los estudios de imagen, la ecocardiografía es la principal modalidad para diagnosticar y valorar la cardiopatía, ya que permite la evaluación de la 
enfermedad valvular, así como del tamaño y la función de la cámara cardíaca derecha. Las técnicas avanzadas, que incluyen TTE 3D y TOE 3D, pueden ayudar a diagnosticar y evaluar la enfermedad de la válvula, especialmente en las válvulas tricúspide y pulmonar, ya que es posible que no se visualicen todas las valvas en la TTE 2D. La tomografía computarizada cardíaca - la resonancia magnética cardíaca pueden complementar a la ecocardiografía cuando se obtienen datos incompletos o cuando las estructuras no se encuentran bien definidas. Los hallazgos ecocardiográficos típicos de la afectación de la válvula tricúspide avanzada incluyen engrosamiento y retracción de las valvas, las cuales son fijas y no coaptan. Esto, generalmente, se asocia con regurgitación tricuspídea grave, perfil Doppler en forma de daga y estenosis tricúspide leve a moderada ${ }^{12}$.

Dentro del tratamiento indicado se encuentran los medicamentos dirigidos a la insuficiencia cardíaca y el tratamiento con análogos de la somatostatina, como por ejemplo telotristat; sin embargo, el reemplazo de la válvula cardíaca es la única terapia definitiva para la cardiopatía coronaria avanzada, y mejora los síntomas y la supervivencia del paciente. No se ha determinado el momento óptimo para la cirugía de reemplazo valvular en la cardiopatía coronaria, pero en general los pacientes con síndrome carcinoide bien controlado y con una esperanza de vida de al menos 12 meses se derivan para realización de cirugía valvular ${ }^{13}$. La elección de la prótesis valvular sigue siendo controvertida, ya que la literatura se limita a estudios retrospectivos, no aleatorizados; pese a ello, esta ha apoyado progresivamente el uso de bioprótesis debido al manejo mejorado del síndrome carcinoide, a las bajas tasas de afectación de carcinoides en series patológicas recientes de bioprótesis, a los resultados favorables a corto plazo, a la probabilidad de que la longevidad de las válvulas bioprotésicas de nueva generación tenga éxito en la supervivencia a mediano y largo plazo del paciente, al riesgo de sangrado inherente en pacientes con metástasis hepáticas y disfunción hepática, y a la probabilidad de cirugía oncológica o quimioterapia en el futuro, para lo cual la anticoagulación a largo plazo puede representar un riesgo adicional. Además, es muy importante el seguimiento posoperatorio de manera multidisciplinaria para determinar la evolución de los pacientes, tanto a nivel clínico como con ecocardiograma de seguimiento para valoración funcional y hemodinámica de las válvulas a corto, mediano y largo plazo $^{14}$.
En el caso que se expone, la paciente fue referida al servicio de cardiología por disnea y se le diagnosticó afectación valvular que le condicionaba signos de insuficiencia cardíaca. Es importante destacar que los pacientes con síndrome carcinoide pueden llegar a tener afectación cardíaca, por lo que se considera conveniente que ante un diagnóstico de tumor carcinoide siempre tengan una valoración por parte de cardiología y se les realice un ecocardiograma transtorácico como tamizaje para poder diagnosticar de manera oportuna el síndrome de Hedinger y establecer el tratamiento quirúrgico, como ocurrió con el caso que se expone, que se encuentra en clase funcional I de la NYHA después de 2 años de su intervención.

\section{Conclusiones}

El síndrome de Hedinger disminuye la supervivencia en los pacientes con tumor carcinoide, por lo cual es de vital importancia realizar un diagnóstico oportuno para poder llevar a cabo el tratamiento quirúrgico y mejorar la calidad de vida.

\section{Financiamiento}

Los autores declaran no haber recibido financiamiento.

\section{Conflicto de intereses}

Los autores declaran no tener conflicto de intereses.

\section{Responsabilidades éticas}

Protección de personas y animales. Los autores declaran que para esta investigación no se han realizado experimentos en seres humanos ni en animales.

Confidencialidad de los datos. Los autores declaran que en este artículo no aparecen datos de pacientes.

Derecho a la privacidad y consentimiento informado. Los autores han obtenido el consentimiento informado de los pacientes y/o sujetos referidos en el artículo. Este documento obra en poder del autor de correspondencia.

\section{Bibliografía} 1. Modlin IM, Lye KD, Kidd M. A 5-decade analysis of 13,715 carcinoid
tumors. Cancer. 2003;97:934-59. 
2. Hayes A, Davar J, Caplin ME. Carcinoid heart disease a review. Endocrinol Metab Clin N Am. 2018;47:671-82.

3. Pellikka PA, Tajik AJ, Khandheria BK, Seward JB, Callahan JA, Pitot HC, et al. Carcinoid heart disease. Clinical and echocardiographic spectrum in 74 patients. Circulation. 1993;87:1188-96.

4. Møller JE, Pellikka PA, Bernheim AM, Schaff HV, Rubin J, Connolly HM. Prognosis of carcinoid heart disease: analysis of 200 cases over two decades. Circulation. 2005;112:3320-7.

5. Yao JC, Hassan M, Phan A, Dagohoy C, Leary C, Mares JE, et al. One hundred years after "carcinoid": epidemiology of and prognostic factors for neuroendocrine tumors in 35,825 cases in the United States. J Clin Oncol. 2008;26:3063-72

6. Warner R, Castillo J. Carcinoid heart disease: the challenge of the unknown known. JACC. 2015;66:2197-200.

7. Castillo JG, Adams DH, Fischer GW. Absence of the tricuspid valve due to severe carcinoid heart disease. Rev Esp Cardiol. 2010;63:96.

8. Grozinsky-Glasberg S, Grossman AB, Gross DJ. Carcinoid heart disease: from pathophysiology to treatment - 'Something in the way it moves'. Neuroendocrinology. 2015;101:263-73.
9. Denney WD, Kemp WE, Anthony LB, Oates JA, Byrd BF $3^{\text {rd }}$. Echocardiographic and biochemical evaluation of the development and progression of carcinoid heart disease. J Am Coll Cardiol. 1998;32:1017-22.

10. Simula DV, Edwards WD, Tazelaar HD, Connolly HM, Schaff HV. Surgical pathology of carcinoid heart disease: a study of 139 valves from 75 patients spanning 20 years. Mayo Clin Proc. 2002;77:139-47.

11. Bruce CJ, Connolly HM. Right-sided valve disease deserves a little more respect. Circulation. 2009;119:2726-34.

12. Bhattacharyya S, Toumpanakis C, Burke M, Taylor AM, Caplin ME, Davar J. Features of carcinoid heart disease identified by two- and three-dimensional echocardiography and cardiac magnetic resonance imaging. Circ Cardiovasc Imaging. 2010;3:103-11.

13. Connolly HM, Schaff HV, Abel MD, Rubin J, Askew JW, Li Z, et al. Early and late outcomes of surgical treatment in carcinoid heart disease. J Am Coll Cardiol. 2015;66:2189-96.

14. El Gabry M, Shehada SE, Mourad F, Ruhparwar A, Lahner H, Dirkmann D, et al. Hedinger syndrome: first experience and two-year follow-up in patients with carcinoid heart disease. J Thorac Dis. 2019;11:3234-40. 